\title{
Odontoma compuesto: reporte caso clínico
}

\section{Compound Odontoma: Clinical case report} \author{
Alexandra' \\ 1 Docente de Odontología en la Universidad Católica de Cuenca. \\ ${ }^{2}$ Estudiante de Odontología en la Universidad Católica de Cuenca. \\ *mayale93@hotmail.es
}

Piedra-Sarmiento Xavier Bernardo;; Moncayo Pinos Jaime Bernardo²; Gonzalez Campoverde Lorena

DOI: https://doi.org/10.26871/killcanasalud.v5i1.823

\section{Resumen}

El odontoma compuesto es una lesión, definida como un hamartoma de tejido odontogénico, es decir una proliferación de células maduras caracterizadas por diversos estados de diferenciación histológica y morfológica que se presenta con una estructura similar a una formación dental. La lesión suele presentarse asintomática y usualmente se diagnostican mediante estudios de radiográficos rutinarios, observándose una lesión radiopaca y radiolúcida aislada de bordes delimitados de morfología similar a una pieza dental. Esta dentro del grupo de los tumores odontogénicos benignos mixtos, de origen ectodérmico y mesodérmico, es indispensable el reconocimiento clínico de la lesión para efectuar el posterior procedimiento quirúrgico.

Se presenta el caso de una paciente femenina de 56 años de edad, la misma acude a consulta con el requerimiento de una prótesis total, alude que tiene una lesión en el cuadrante superior derecho que le causa molestias al momento de ingerir alimentos con su prótesis actúa, es por este motivo que se llevan a cabo exámenes radiográficos, en los mismos fue identificado una lesión radiopaca extraosea, se identifica la lesión como un odontoma en la región edéntula del cuadrante 2, el mismo que fue removido mediante un procedimiento quirúrgico en el mismo se pudo identificar un odontoma compuesto.

Palabras Clave: Odontoma compuesto, Tumor odontogénico. 


\section{Abstract}

The Compound odontoma is a lesion, which is defined as a hamartoma of odontogenic tissue, that is to say, a proliferation of mature cells characterized by various stages of histological and morphological differentiation that is similar in structure to the dental formation. The lesion is usually asymptomatic and is diagnosed by routine radiographic studies. It is seen as an isolated radio opaque and radio lucid lesion with delimited borders and a morphology similar to a dental piece. It is classified as a benign mixed odontogenic tumor of ectodermal and mesodermal origin. Clinical diagnosis of the lesion is essential for the subsequent surgical procedure.

The following case is presented. A 56-year-old female patient came for consultation requiring a total prosthesis, she mentioned having a lesion in the right upper quadrant that causes her discomfort when eating with her current prosthesis. Because of this, radiographic examinations were performed, in which an extra oseous radiopaque lesion was observed. The lesion was identified as an odontoma in the edentulous region of quadrant 2 , which was removed by a surgical procedure, identifying a compound odontoma.

Palabras Clave: Compound odontoma, Odontogenic tumor. 


\section{Introducción}

En 1867 Broca define al odontoma como un tumor benigno de origen odontogénico, considerado como una lesión de células epiteliales y mesenquimatosas, diferenciadas que forman esmalte, dentina y cemento. Esta neoplasia representa el $51 \%$ de todos los tumores odontogénicos siendo así los de mayor frecuencia de aparición. ${ }^{1-4}$

Los odontomas suelen ser clínicamente asintomáticos, de crecimiento limitado, producen retención dentaria y su hallazgo es casual en revisiones radiográficas rutinarias. Cuando presentan alguna sintomatología, los signos clínicos más frecuentes son el retraso en la erupción dental y la tumefacción a nivel del proceso alveolar afectado. ${ }^{4-6}$

La etiología esta relacionada con restos paradentales de malassez, traumatismos, infecciones, mutaciones genéticas (síndrome de Hermman, enfermedad de Tangier, nevus de células basales, el síndrome de Gardner y la adenomatosis colónica familiar), la hiperactividad odontoblástica 0 alteraciones en el gen de control del desarrollo dentario. ${ }^{4}$ La Organización Mundial de la Salud (OMS) clasifica a los odontomas en compuestos y complejos. En el compuesto se presentan todos los tejidos dentarios, formando una lesión con múltiples estructuras de aspecto dentario, el complejo se presentan todos los tejidos dentarios pero siguiendo un patrón más desordenado. ${ }^{1-6}$

El odontoma compuesto es más frecuente que el complejo y la mayoría se presentan en la segunda década de la vida. Con predilección por el sexo masculino. Habitualmente están en el sector anterior derecho del maxilar superior. ${ }^{2,3}$

Desde el punto de vista histopatológico estos tejidos y células se encuentran en el área del cuerpo donde se presenta su crecimiento, razón por la cual se les considera como hamartomas y no como neoplasias, el odontoma compuesto presenta pulpa, dentina primaria, esmalte hipocalcificado, cemento primario y un trabeculado óseo entre los dentículos. Existen formas mixtas con morfología intermedia. ${ }^{2-5}$

Radiográficamente el odontoma compuesto se presenta como una imagen radiopaca y radiolúcida, que adopta morfología similar a la dental (dentículos), rodeados por un halo radiolúcido en más del $50 \%$ de los casos. ${ }^{6-7}$

En su mayoría, los odontomas son asintomáticos, en el caso que se presente sintomatología, esta se relaciona con su presencia, dientes permanentes impactados $(57,7 \%)$, retención de dientes deciduos $(16,6 \%)$, desplazamiento de dientes adyacentes $(11,1 \%)$, expansión de corticales óseas $(8,9 \%)$ y una perdida congénita del diente $(3,3 \%)$. 2,7,8

El objetivo de este estudio es describir al odontoma compuesto a través de una revisión bibliográfica y presentación de un caso clínico, estudiado a través del diagnóstico radiográfico y su tratamiento quirúrgico. 


\section{Caso clínico}

Paciente de 56 años de edad del sexo femenino, acude a la clínica de Odontología de la Universidad Católica de Cuenca, el motivo de consulta fue la necesidad de una prótesis total superior. Al examen clínico intraoral se observa que en el cuadrante alveolar superior izquierdo presenta aumento de tamaño del reborde (Fig. 1), acompañado de una masa ulcerada (Fig.2) y sólida a la palpación. (Figura 1 y 2). La paciente refiere que la lesión le causa molestias al momento de ingerir alimentos y que la misma impedía una correcta adaptación de su prótesis antigua.
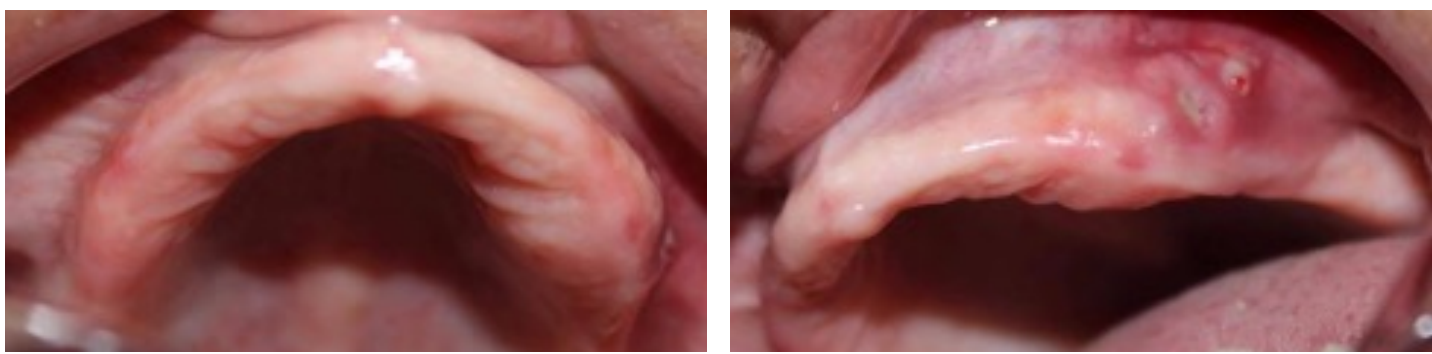

Fig. 1 y 2: Vista oclusal y lateral de la lesión.

Se realizó una radiografía panorámica y periapical (Figura 2-3), para la valoración de la lesión, llegando al diagnóstico presuntivo de un odontoma, debido a que se presenta como una lesión radiopaca de bordes delimitados (Fig. 3) y de densidades similares a las de una pieza dental (Fig. 4).

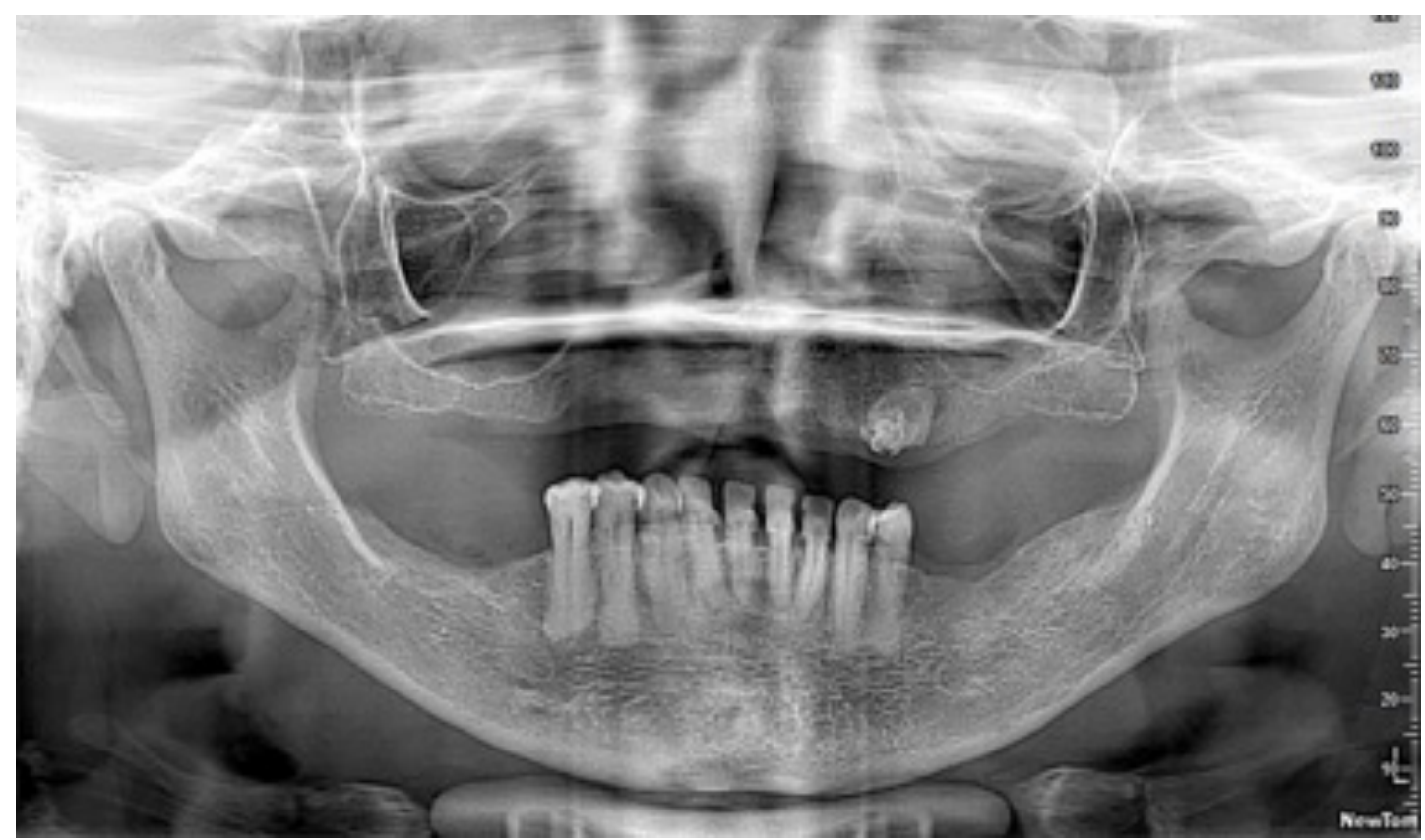

Fig. 3: Lesión radiopaca aislada en el cuadrante superior derecho 


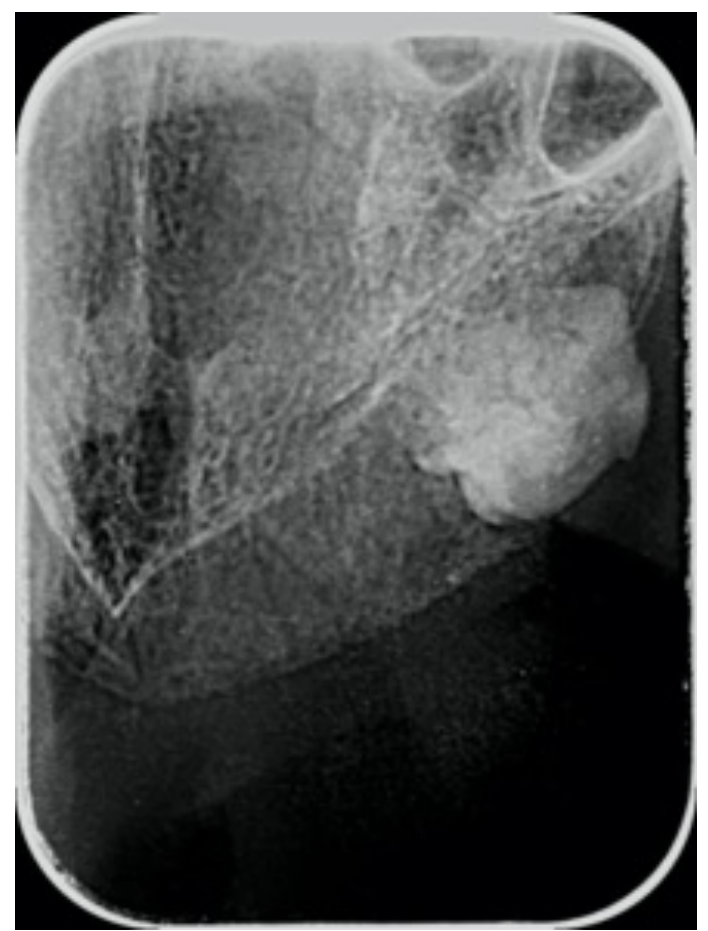

Fig. 4: Radiografía periapical oclusal muestra una lesión extra-ósea aislada

Como fue descrito por la paciente la lesión causaba molestias y se observa que el reborde aumentado de tamaño (Fig. 1 y 2) comprometiendo la adaptabilidad de la prótesis nueva, es por este motivo que se llevó a cabo el tratamiento quirúrgico.

Luego de la asepsia y antisepsia de la zona se procede a anestesiar, mediante una incisión triangular (Fig. 5), con una elevación de colgajo mucoperióstico (Fig. 6), el mismo permitió observar la presencia de elementos compatibles con teidos dentarios (Fig. 7), fue necesario llevar a cabo una osteotomía que permitió liberar la tumoración en la que se visualiza con precisión la similitud con una pieza dental (Fig. 8) llegando al diagnóstico intraquirúrgico de odontoma compuesto, el mismo que fue removido mediante una incisión del alveolo; el procedimiento finalizó con la regularización del reborde alveolar (Fig. 9) y sutura (Fig. 10).

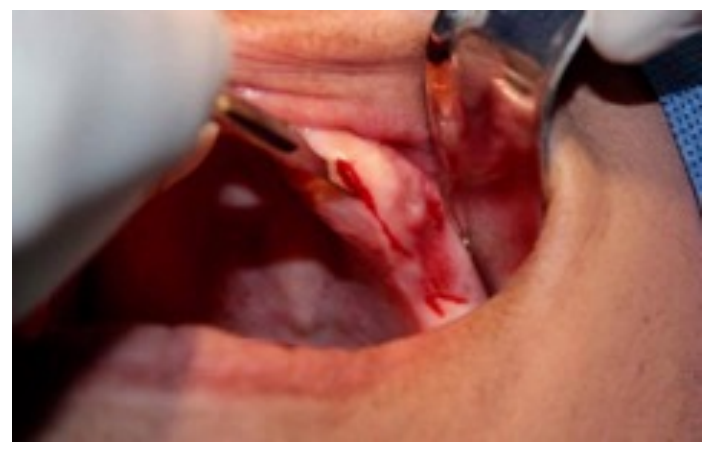

Fig 5. Incisión

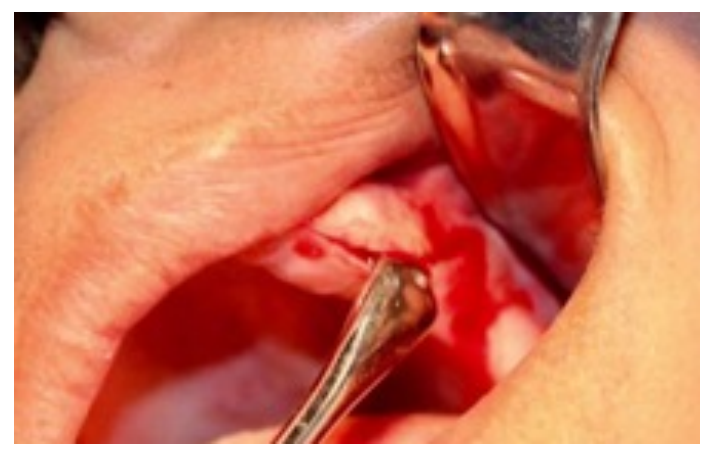

Fig 6. Sindesmotomía 


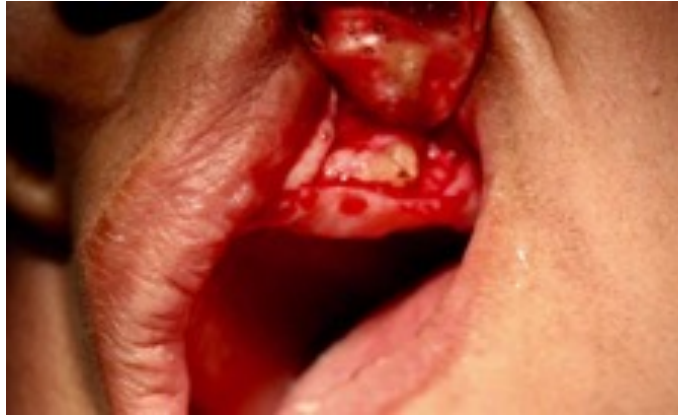

Fig 7. Visualización de la lesión

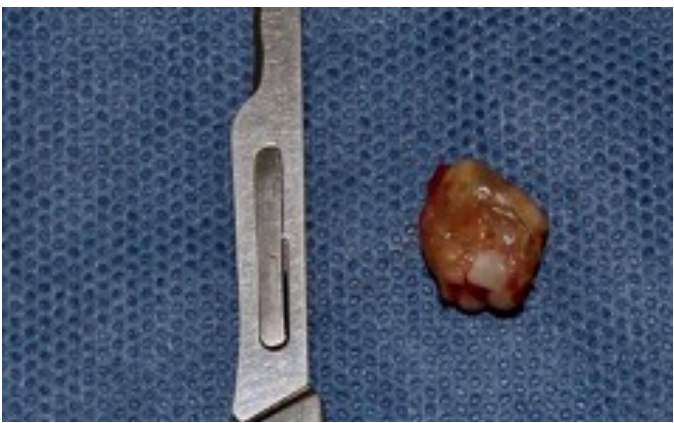

Fig 9. Regularización del reborde alveolar

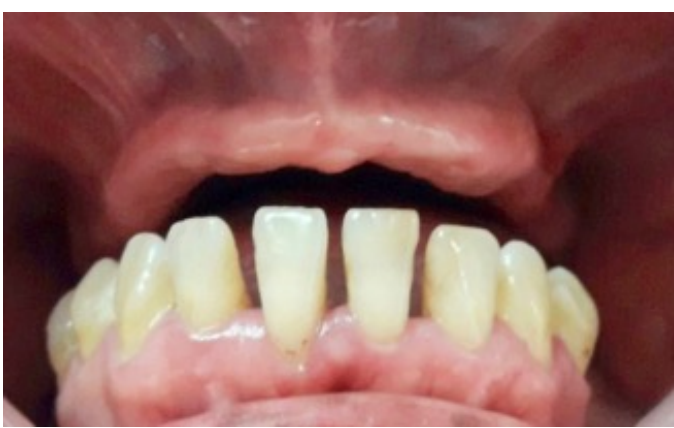

Fig 11. Posoperatorio 3 meses después

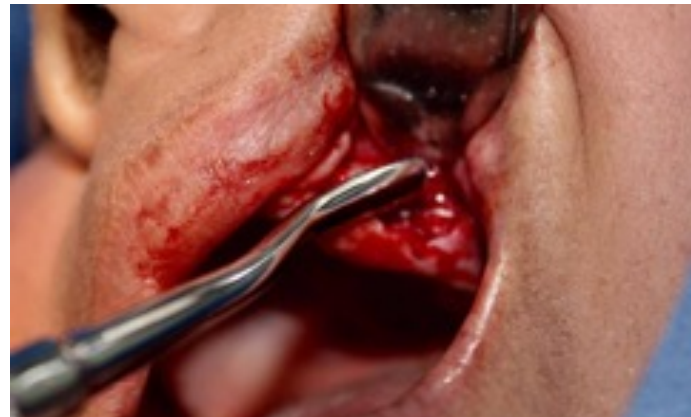

Fig 8. Odontoma compuesto de 9x7x6 mm

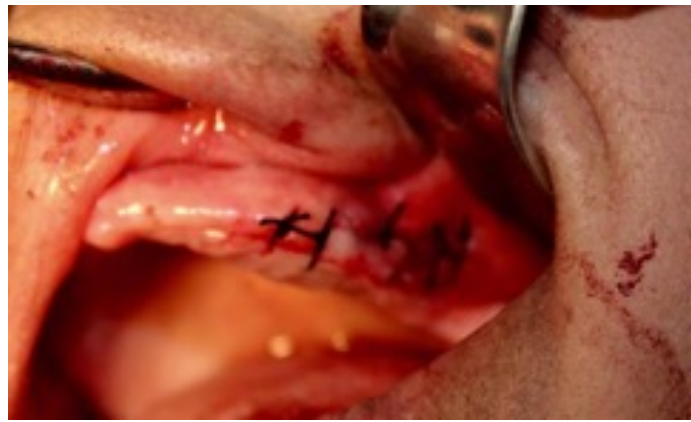

Fig 10. Sutura

Siete días después de efectuado el procedimiento se observa una recuperación sin complicaciones y se procede a retirar las suturas.

El control postoperatorio se llevo a cabo tres meses después del procedimiento, muestra una recuperación completa mostrando un reborde regular (Fig. 11). Mediante una radiografía periapical oclusal se puede observar la enucleación completa de la lesión (Fig. 12). 


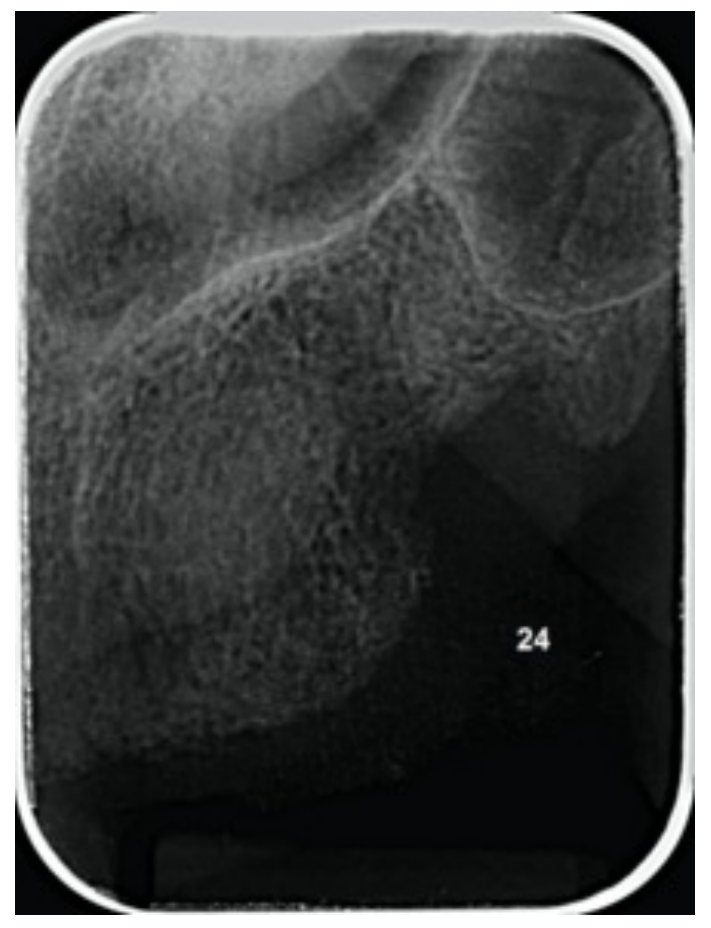

Fig. 12. Radiografia periapical oclusal 3 meses después.

\section{Discusión}

Los odontomas son tumores benignos que se observan con frecuencia en la cavidad oral, se presentan como asintomáticos y son hallazgos casuales de estudios radiográficos rutinarios, como en el caso reportado. Cuando causan sintomatología se puede observas retraso en la erupción dental o un reborde aumentado de tamaño. El diagnóstico final se realiza mediante exámenes complementarios. ${ }^{2-4}$

Radiográficamente se puede observar a los odontomas como con una lesión radiopaca densa rodeada por un halo radiotransparente delgado. ${ }^{3-6}$

El diagnóstico radiográfico de un odontoma debe ser diferenciado con lesiones de localización entre raíces como osteítis residual focal, tumores odontogénicos epiteliales calcificacantes, cementoma, tumores ontogénicos adenomatoides, dientes supernumerarios, fibroma cementante o un osteoblastoma benigno, asi tenemos que si la lesión está localizada en el periodonto debe diferenciarce con un tumor odontogénico adenomatoide, tumores odontogénicos epiteliales calcificantes, odontoameloblastoma o fibrodentinoma ameloblástico. Si la lesión se ubica en el seno maxilar puede ser confundida con sinusitis, infección periapical con pólipo antral, neoplasma mesenquimatoso benigno, papiloma inversus o carcinoma. ${ }^{10}$

Histológicamente los odontomas compuesto constan de un saco de tejido fibroso conectivo que rodea un dentículo, un dentículo está compuesto por un núcleo con tejido similar a la pulpa dental, rodeado de dentina primaria y cubierto con esmalte que se encuentra desmineralizado y cemento primario. ${ }^{10-11}$ 
Los odontomas complejos a su vez dejan ver dentina inmadura como componente predominante, suelen además presentar esmalte, con 2 posibles tipos de distribución: un área calcificada cerca del núcleo central, o en una región hipocalcificada con esmalte inmaduro, además es posible observar cemento inmaduro, con la cápsula de tejido conectivo que rodea la lesión. ${ }^{12}$

Los odontomas pueden causar expansión ósea ligera, en cambió los odontomas compuestos causan gran distensión ósea, han sido asociados a situaciones como mal posición dental, impactación o mal posición, así como la formación, reabsorción y la desvitalización de dientes adyacentes en el $70 \%$ de los casos.

La mayoría de autores reportan los odontomas en pacientes jóvenes con afectación de tejidos blandos, a diferencia del presente caso clínico la lesión la presento una paciente adulta mayor, en el caso del maxilar la mayoría de lesiones se ubican en la región anterior, creando compromiso estético. $(8,11-12)$

El diagnóstico como refiere la bibliografía fue ocasional por medio de una radiografía panorámica se pudo observar una lesión con características de un odontoma compuesto, se llevo a cabo una técnica mínimamente invasiva para la extirpación de la lesión la misma se confirmó el diagnóstico radiográfico la radiografía del posoperatorio presenta una recuperación completa asi como la devolución de la funcionalidad y estética al paciente.

A partir de la observación de las imágenes de control post operatorio, consideramos que la aplicación de la técnica elegida para el tratamiento de la lesión fue la adecuada para este caso particular y que, sumada a un tratamiento de ortodoncia, brindará a la paciente un resultado funcional y estético óptimo. 


\section{Referencias Bibliográficas:}

1. Dávila-Hernández DA. Enucleación de un odontoma compuesto mandibular. Comunicación de un caso clínico. Rev Esp Med Quir 2010;15(2):92-96. Disponible en: http://www. medigraphic.com/pdfs/quirurgicas/rmq-2010/rmq102h.pdf

2. Peranovic V, Noffke CEE. Clinical and radiological features of 90 odontomas diagnosed in the Oral Health Centre at Sefako Makgatho Health Sciences University. S. Afr. dent. j. [Internet]. 2016 Nov; 71( 10 ): 489-492. Disponible en: http://www.scielo.org.za/scielo. php?script=sci_arttext\&pid=S0011-85162016001000011\&Ing=en

3. Vázquez Diego J, Gandini Pablo C, Carbajal Eduardo El Odontoma compuesto: Diagnóstico radiográfico y tratamiento quirúrgico de un caso clínico 2008. Disponible en: http:// scielo.isciii.es/pdf/odonto/v24n5/original1.pdf

4. Barnes $L$, eveson JW, reichart $P$, Sidransky $D$ (eds.). Pathol- ogy and Genetics of head and Neck tumours. Lyon: IArc Press; 2005:284

5. Jonathan Harris R, Diaz A y Carbonell Z. Odontoma compuesto Una patología oral a considerar. Duazary; Santa Marta 7.1 (Jan-Jun 2010): 91-94. Disponible en: http://search. proquest.com/openview/7d39c6cbf22e9c0dcbead53b1d993f20/1?pq-origsite=gscholar\&cbl=2043249

6. Guerra Treviño A. y Cols. Odontoma compuesto: Diagnóstico radiológico y tratamiento quirúrgico de un caso. Revista adm/mayo-junio 2012/ vol. Lxix no. 3. P.p. 139-141. Disponible en: http://www.medigraphic.com/pdfs/adm/od-2012/od123i.pdf

7. Harris Ricardo J, Rebolledo Cobos M, Díaz Caballero A, Carbonell Muñoz Z. Odontoma serie de casos. Revisión de literatura. Av. Odontoestomatol 2011; 27(1): 25-32. Disponible en: http://scielo.isciii.es/pdf/odonto/v27n1/original2.pdf

8. Brenes J.“Odontoma Compuesto: Diagnóstico Radiográfico y Manejo Quirúrgico. Reporte de dos casos clínicos" caso clínico. REV. CIENT. ODONTOL. (9) 2: 39-43 2013 Disponible en : http://www.colegiodentistas.org/revista/index.php/revistaodontologica/article/ viewFile/217/327

9. Amado-Cuesta S, Gargallo-Albiol J, Berini-Aytés L, Gay-Escoda C. Revisión de 61 casos de odontoma. Presentación de un odontoma complejo erupcionado. Med Oral 2003;8:36673 http://diposit.ub.edu/dspace/bitstream/2445/103351/1/513860.pdf

10. Ide F, Shimoyama T, Horie N. Gingival peripheral odontoma in an adult: case report. J Periodontol 2000;71:830-2. http://onlinelibrary.wiley.com/doi/10.1902/jop.2000.71.5.830/ abstract;jsessionid=70AFC1B35B3EA02965967C8DA3D56D94.f02t01

11. Kaneko M, Fukuda M, Sano T, Ohnishi T, Hosokawa Y. Microradiographic and microscopic investigation of a case of complex odontoma. Oral Surg Oral Med Oral Pathol Oral Radiol Endod 1998;85:131-4. Disponible en: https://www.sciencedirect.com/science/article/ pii/S1079210498901639

12. Garcia Rivera ME y Cols. Odontoma Compuesto: Reporte de un Caso Revista Médica de la Universidad Veracruzana / Vol.15, no. 2, julio-diciembre de 2015. Disponible en: https:// www.uv.mx/rm/num_anteriores/revmedica_vol15_num2/articulos/odontoma.pdf 
13. Sikes JW, Ghali GE, Troulis MJ. Expansile intraosseous lesion of the maxilla. J Oral Maxillofac Surg 2000;. . http://www.joms.org/article/S0278-2391(08)00267-X/fulltext

Recibido: 08 Septiembre 2020

Aceptado: 15 Diciembre 2020 